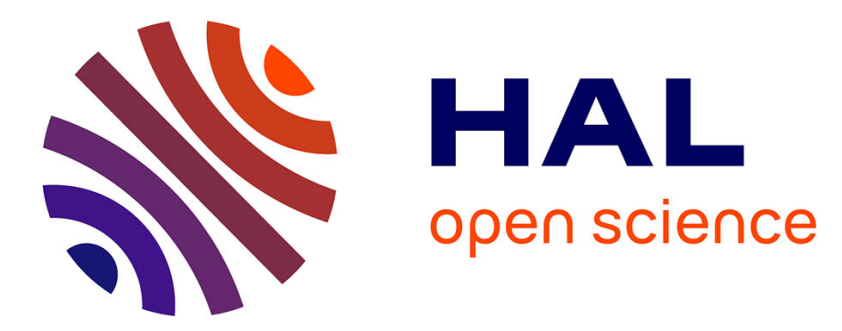

\title{
Bending and buckling of inflatable beams : some new theoretical results
}

\author{
Anh Le Van, Christian Wielgosz
}

\section{To cite this version:}

Anh Le Van, Christian Wielgosz. Bending and buckling of inflatable beams : some new theoretical results. Thin-Walled Structures, 2005, 43 (8), pp.1166-1187. 10.1016/j.tws.2005.03.005 . hal-01005290

\section{HAL Id: hal-01005290 \\ https://hal.science/hal-01005290}

Submitted on 15 Oct 2016

HAL is a multi-disciplinary open access archive for the deposit and dissemination of scientific research documents, whether they are published or not. The documents may come from teaching and research institutions in France or abroad, or from public or private research centers.
L'archive ouverte pluridisciplinaire HAL, est destinée au dépôt et à la diffusion de documents scientifiques de niveau recherche, publiés ou non, émanant des établissements d'enseignement et de recherche français ou étrangers, des laboratoires publics ou privés. 


\title{
Bending and buckling of inflatable beams: Some new theoretical results
}

\author{
A. Le van, C. Wielgosz \\ Faculty of Sciences of Nantes, GeM Institute of Research in Civil and Mechanical Engineering, \\ 2, rue de la Houssinière, BP 92208, Nantes 44322 cedex 3, France
}

The non linear and linearized equations are derived for the in plane stretching and bending of thin walled cylindrical beams made of a membrane and inflated by an internal pressure. The Timoshenko beam model combined with the finite rotation kinematics enables one to correctly account for the shear effect and all the non linear terms in the governing equations. The linearization is carried out around a pre stressed reference configuration which has to be defined as opposed to the so called natural state. Two examples are then investigated: the bending and the buckling of a cantilever beam. Their analytical solutions show that the inflation has the effect of increasing the material properties in the beam solution. This solution is compared with the three dimensional finite element analysis, as well as the so called wrinkling pressure for the bent beam and the crushing force for the buckled beam. New theoretical and numerical results on the buckling of inflatable beams are displayed.

Keywords: Inflatable beam; Thin walled beam; Membrane structure; Follower force; Bending; Buckling

\section{Introduction}

There are only a few papers dealing with theoretical studies on inflatable beams and it seems that this research subject has been neglected in the last years. The first paper by Comer and Levy [1] was published about 40-years-ago and its aim was to prove that 
inflatable beams can be considered as usual Euler Bernoulli beams. At the same time, Fichter [2] published a very interesting paper in which deflection of inflatable beams is obtained by means of the principle of the total potential energy minimum. By taking into account large displacements and following forces, Fichter has successfully included the internal pressure in the equilibrium equations for inflatable beams subjected to bending and compressive loads. Main et al. [3] realized experiments on cantilever inflatable beams and made use of Comer and Levy's theory. The pressure range considered in their experiments was not significant enough to reveal the influence of the inflation pressure, and their conclusion was that the usual Euler Bernoulli beam theory introduced by Comer and Levy can be applied. However, as shown in [4,5], a usual beam theory cannot be used to estimate the deflection of inflatable beams, because the inflation pressure does not appear in the expression for the deflection.

In two recent papers, dealing with the mechanics of inflatable beams, one of the authors has shown that the pressure effects should be involved in the final deflection, thus reinforces Fichter's solution. Deflections of flat panels [4] and tubes [5] have been obtained under the following assumptions: the equilibrium equations should be written in the deformed configuration in order to take into account the follower loads; Timoshenko's theory should be used to describe the kinematics of the beam (see Fig. 5 in [4] and Fig. 2 in [5]). Beam analytical and finite element solutions [6] were obtained to give the sought deflection. In every case, comparisons between theoretical solutions and experimental results for simply supported inflated beams with various boundary conditions have shown a very good agreement.

In the present work, a new formulation for inflatable beams is proposed in order to improve Fichter's theory. The non-linear equations for the in-plane stretching and bending of an inflated beam are derived from the Lagrangian form of the virtual work principle. One additional assumption compared with Fichter's ones is introduced: the finite rotation kinematics, which is shown to enable one to correctly account for all the non-linear terms in the governing equations. Subsequently, the whole equation set is linearized around a pre-stressed reference configuration which has to be defined as opposed to the so-called natural state. Two examples are then investigated: the bending and the buckling of a cantilever beam. Their analytical solutions show that the inflation has the effect of 'increasing the material properties.' The numerical results are compared with the membrane finite element ones obtained within the three-dimensional framework. Eventually, discussion of the validity of the solutions exhibits the notions of the wrinkling pressure for the bent beam and the crushing force for the buckled beam. To the authors' knowledge, the results on the buckling of inflatable beams are novel.

\section{Governing equations}

Consider an inflatable beam made of a cylindrical membrane undergoing axial stretch and bending in the $x y$-plane (Fig. 1) under the combined action of an internal pressure and other external dead loads. In the reference (or initial) configuration, the length of the beam is $l_{0}$, the cross-section area $S_{0}$, the second moment of area $I_{0}$, and all the centroids $G_{0}$ of the cross-sections lie on the $x$-axis. 


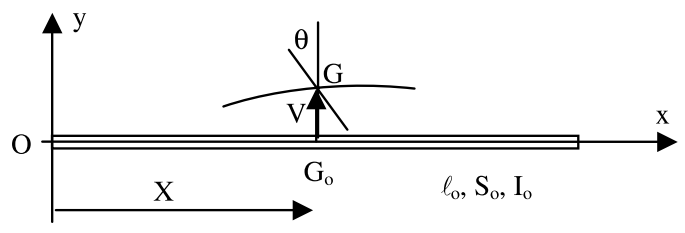

Fig. 1. The inflated beam model.

In order to derive the governing equations for the inflatable beam, use will be made of the principle of virtual work in the three-dimensional Lagrangian form

$$
\begin{gathered}
\forall \text { virtual displacement field } \mathbf{V}^{*}, \quad \int_{\Omega_{0}} \Pi^{\mathrm{T}}: \operatorname{grad} \mathbf{V}^{*} \mathrm{~d} \Omega_{0}+\int_{\Omega_{0}} \mathbf{f}_{0} \cdot \mathbf{V}^{*} \mathrm{~d} \Omega_{0} \\
+\int_{\partial \Omega_{0}} \mathbf{V}^{*} \cdot \Pi \cdot \mathbf{N} \mathrm{d} S_{0} \quad 0
\end{gathered}
$$

where $\Omega_{0}$ is the region occupied by the body in the reference configuration, $\partial \Omega_{0}$ its boundary, $\Pi$ the first Piola Kirchhoff stress tensor, $\mathbf{f}_{0}$ the body force per unit reference volume and $\mathbf{N}$ the unit outward normal in the reference configuration.

\subsection{Displacement and strain fields}

Let us denote by $\mathbf{U}(X) \quad(U(X), V(X), 0)$, the displacement of the centroid of a current cross-section at abscissa $X$ (all the components are related to the base $(\mathbf{x}, \mathbf{y}, \mathbf{z})$ ). We assume that during the deformation the cross-section remains plane, but not perpendicular to the bent axis of the beam (Timoshenko beam model). Then, by denoting $\theta(X)$ the finite rotation of the cross-section, the displacement of any material point $\mathbf{P}_{0}(X, Y, Z)$ is given by:

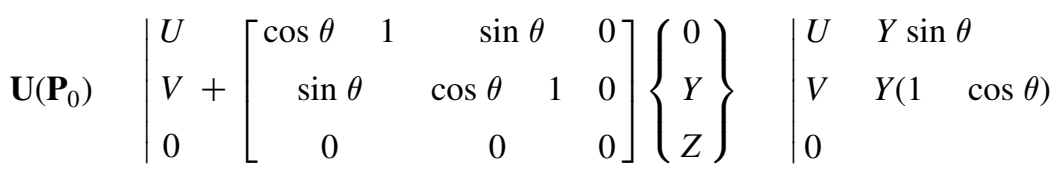

One readily deduces the components of the Green strain tensor from (2):

$$
\begin{aligned}
& E_{X X} \quad U_{, x} \quad Y \cos \theta \theta_{, x}+\frac{1}{2}\left[U_{, x}^{2}+V_{, x}^{2}+Y^{2} \theta_{, x}^{2} \quad 2 Y U_{, x} \cos \theta \theta_{, x}\right. \\
& \left.2 Y V_{, x} \sin \theta \theta_{, x}\right] \\
& E_{X Y} \quad \frac{1}{2}\left[\begin{array}{llll}
V_{, x} \cos \theta & \left(1+U_{, x}\right) \sin \theta
\end{array}\right] \quad E_{Y Y} \quad 0
\end{aligned}
$$

The axial strain $E_{X X}$ involves linear and quadratic terms in $Y$, whereas the shear component $E_{X Y}$ does not depend on $Y$ and is constant over the cross-section. 


\subsection{Virtual displacement field}

Let us denote by $\mathbf{V}^{*}(X) \quad\left(U^{*}(X), V^{*}(X), 0\right)$, the virtual displacement of the centroid of a current cross-section. The following expression is chosen for the virtual displacement of a current material point $\mathbf{P}_{0}$

$$
\mathbf{V}^{*}\left(\mathbf{P}_{0}\right) \quad \mathbf{V}^{*}+\Omega^{*} \times \mathbf{G} P
$$

where $\Omega^{*}(X) \quad\left(0,0, \theta^{*}(X)\right)$ is the virtual rotation. Note that Relation (4) involves the final vector $\mathbf{G P}$, not the initial vector $\mathbf{G}_{0} \mathbf{P}_{0}$, as in the real velocity field in mechanics of rigid bodies. Since vector $\mathbf{G P}$ in turn can be expressed in terms of Lagrangian variables by using the rotation operation: GP ( $\mathrm{Y} \sin \theta, \mathrm{Y} \cos \theta, Z)$, it comes from (4):

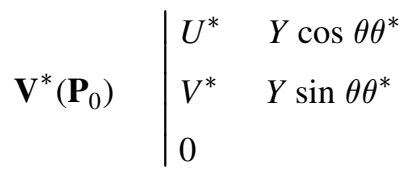

Thus, the virtual displacement field is completely defined by three scalar functions $U^{*}(X), V^{*}(X)$ and $\theta^{*}(X)$.

\subsection{Stresses}

The matrix of the second Piola Kirchhoff (symmetric) stress tensor $\Sigma$ in the base $(\mathbf{x}, \mathbf{y}, \mathbf{z})$ is assumed to take the following form:

$$
[\Sigma] \quad\left[\begin{array}{ccc}
\Sigma_{X X} & \Sigma_{X Y} & 0 \\
\Sigma_{X Y} & 0 & 0 \\
0 & 0 & 0
\end{array}\right]
$$

The material is assumed to be hyperelastic isotropic, obeying the Saint Venant Kirchhoff constitutive law, characterized by the Young modulus $E$, the Poisson ratio $\nu$ and the initial (or residual) stresses $\Sigma_{X X}^{0}, \Sigma_{X Y}^{0}$ induced by the preliminary inflation of the beam:

$$
\Sigma_{\mathrm{XX}} \quad \Sigma_{\mathrm{XX}}^{0}+E \cdot E_{\mathrm{XX}} \quad \Sigma_{\mathrm{XY}} \quad \Sigma_{\mathrm{XY}}^{0}+2 G \cdot E_{\mathrm{XY}}\left(\begin{array}{ll}
G & \left.\frac{E}{2(1+\nu)}\right)
\end{array}\right.
$$

In the sequel, it is convenient to introduce the following generalized stresses

$$
N \quad \int_{S_{0}} \Sigma_{X X} \mathrm{~d} S_{0} \quad T \int_{S_{0}} \Sigma_{X Y} \mathrm{~d} S_{0} \quad M \quad \int_{S_{0}} Y \Sigma_{X X} \mathrm{~d} S_{0}
$$

which represent the (material) axial force, the shear force and the bending moment acting on the reference cross-section $S_{0}$. By assuming that the cross-section is symmetrical with respect to the $G_{0} \mathbf{z}$-axis so that $\int_{S_{0}} Y \mathrm{~d} S_{0} \quad 0$ and $\int_{S_{0}} Y^{3} \mathrm{~d} S_{0} \quad 0$, one gets the expressions 
for the generalized stresses in terms of the displacements from (3), (7) and (8):

$$
\begin{array}{ll}
N & N^{0}+E S_{0}\left(U_{, X}+\frac{1}{2} U_{, X}^{2}+\frac{1}{2} V_{, X}^{2}+\frac{I_{0}}{2 S_{0}} \theta_{, X}^{2}\right) \\
T & T^{0}+G S_{0}\left(V_{, X} \cos \theta \quad\left(1+U_{, X}\right) \sin \theta\right) \\
M & M^{0}+E I_{0}\left(\left(1+U_{, X}\right) \cos \theta+V_{, X}, X, X \sin \theta\right) \theta_{, X}
\end{array}
$$

In the above, $N^{0}, T^{0}$ and $M^{0}$ are the resultants of the initial stresses on the cross-section. As is seen later, very often simplifying assumptions on $\Sigma_{X X}^{0}, \Sigma_{X Y}^{0}$ lead to $T^{0} M^{0} 0$. Furthermore, in practice the coefficient $G S_{0}$ in (9) is replaced by $k G S_{0}$, where the so-called correction shear coefficient $k$ is determined from the shape of the cross-section. The value usually found in the literature (see, e.g. Ref. [7]) for circular thin tubes is $k \quad 0.5$.

\subsection{Virtual stress work}

From the relation between the first and second Piola Kirchhoff stresses $\Pi \quad \mathbf{F} \Sigma(\mathbf{F}$ is the deformation gradient) and the definitions (8), we obtain the expression for the internal virtual work:

$$
\begin{aligned}
\int_{\Omega_{0}} \Pi^{\mathrm{T}}: \operatorname{grad} \mathbf{V}^{*} \mathrm{~d} \Omega_{0} \\
\\
\quad \int_{0}^{I_{0}}\left[\left(N\left(1+U_{, x}\right)+M \cos \theta \theta_{, x} \quad T \sin \theta\right)\right] U_{, X}^{*}+\left[\left(N V_{, x}+M \sin \theta \theta_{, x}\right.\right. \\
\quad+T \cos \theta)] V_{, X}^{*}+\left[M\left(\quad\left(1+U_{, x}\right) \sin \theta \theta_{, x}+V_{, x} \cos \theta \theta_{, x}\right) \quad T((1\right. \\
\left.\left.\left.+U_{, x}\right) \cos \theta+V_{, x} \sin \theta\right)\right] \theta^{*} \\
\quad+\left[M\left(\left(1+U_{, x}\right) \cos \theta+V_{, x} \sin \theta\right)+\int_{S_{0}} Y^{2} \Sigma_{, X X} \mathrm{~d} S_{0} \theta_{, x}\right] \theta^{*}{ }_{, x} \mathrm{~d} X
\end{aligned}
$$

The integral $\int_{S_{0}} Y^{2} \Sigma_{, X X} \mathrm{~d} S_{0}$ in (10) can be recast as follows, assuming that the initial stress $\Sigma_{X X}^{0}$ takes the following form general enough in practical purposes: $\Sigma_{X X}^{0} \quad \alpha^{0}+$ $\beta^{0} Y+\gamma^{0} Y^{2}$ and that $\Sigma_{X Y}^{0}$ does not depend on $Y$. Hence, from (3a) and (7a) $\Sigma_{X X}$ is of the form $\Sigma_{X X} \quad \alpha+\beta Y+\gamma Y^{2}$, where $\gamma \quad \gamma^{0}+(1 / 2) E \theta_{, x}^{2}$. Substituting this into definition (8) gives

$$
N \quad \int_{S_{0}} \Sigma_{X X} \mathrm{~d} S_{0} \quad \alpha S_{0}+\gamma I_{0} \quad M \quad \int_{S_{0}} y \Sigma_{X X} \mathrm{~d} S_{0} \quad \beta I_{0}
$$


which entails

$$
\Sigma_{\mathrm{XX}} \quad \frac{N}{S_{0}} \quad \frac{\gamma I_{0}}{S_{0}} \quad \frac{M}{I_{0}} Y+\gamma Y^{2}
$$

and then

$$
\int_{S_{0}} Y^{2} \Sigma_{, X X} \mathrm{~d} S_{0} \quad \frac{N I_{0}}{S_{0}}+\gamma\left(\int_{S_{0}} Y^{4} \mathrm{~d} S_{0} \quad \frac{I_{0}^{2}}{S_{0}}\right) \quad \frac{N I_{0}}{S_{0}}+\frac{1}{2} K E \theta_{, x}^{2}+K \gamma^{0}
$$

where

$$
K \quad \int_{S_{0}} Y^{4} \mathrm{~d} S_{0} \quad \frac{I_{0}^{2}}{S_{0}}
$$

is a quantity depending on the initial geometry of the cross-section, like $S_{0}$ and $I_{0}$. This quantity is involved in the non-linear equations of the problem; however, it will be seen that it disappears in the linearized theory.

\subsection{External virtual work due to dead loads}

The Lagrangian expression for the external virtual work due to dead loads is:

$$
W_{\text {ext }}^{*} \quad \int_{\Omega_{0}} \mathbf{f}_{0}\left(\mathbf{P}_{0}\right) \cdot \mathbf{V}^{*}\left(\mathbf{P}_{0}\right) \mathrm{d} \Omega_{0}+\int_{\partial \Omega_{0}} \mathbf{V}^{*}\left(\mathbf{P}_{0}\right) \cdot \Pi\left(\mathbf{P}_{0}\right) \cdot \mathbf{N}\left(\mathbf{P}_{0}\right) \mathrm{d} S_{0}
$$

Since the handling of dead load work is standard, it will not be detailed here. Let us just give its final Eulerian expression in the case of the beam:

$$
\begin{aligned}
W_{\mathrm{ext}}^{*} & \int_{0}^{l}\left(p_{x} U^{*}+p_{y} V^{*}+\mu \theta^{*}\right) \mathrm{d} x+X(0) U^{*}(0)+Y(0) V^{*}(0)+\Gamma(0) \theta^{*}(0) \\
& +X(I) U^{*}(I)+Y(I) V^{*}(I)+\Gamma(I) \theta^{*}(I)
\end{aligned}
$$

In the above, $l$ is the current length, $\left(p_{x}, p_{y}\right)$ are the in-plane components of the dead load per unit length, $\mu$ the moment per unit length, $(X(\cdot), Y(\cdot))$ designates the components of the resultant force at end sections $x \quad 0$ or $l, \Gamma$ the resultant moment. All these loads are applied in the current configuration. It should be mentioned that expression (14) is obtained since vector GP is involved in the virtual displacement (4) instead of $\mathbf{G}_{0} \mathbf{P}_{0}$.

\subsection{External virtual work due to the internal pressure}

Before external dead loads are applied, the beam is inflated by an internal pressure $p \cdot \mathbf{n}$ (where $\mathbf{n}$ is the outward unit normal in the current configuration) which gives the beam its bearing capacity.

The contribution of the internal pressure will be determined under the assumption that the reference volume $\Omega_{0}$ is a circular cylinder of radius $R_{0}$. This assumption, combined with the Timoshenko kinematics (2), implies that any cross-section remains a circular disk of radius $R_{0}$ 
during the deformation. This means that the change in shape (ovalization or warping) of the cross-section is not taken into account, as is the case of all usual beam models.

The virtual work of the pressure is computed by adding the works on the cylindrical surface and on both ends.

\subsubsection{Integral over the cylindrical surface}

Let us represent the reference cylindrical surface by curvilinear coordinates $\left(\xi_{1}, \xi_{2}\right)$ $\left(R_{0} \varphi, X\right)$, where $\varphi$ is the polar angle between the normal $\mathbf{n}$ at the point $\mathbf{x}$ with the $y$-axis. According to the displacement field (2), the current element surface is:

$$
\begin{aligned}
& \mathbf{n d} S \frac{\partial \mathbf{x}}{\partial \xi_{1}} \frac{\partial \mathbf{x}}{\partial \xi_{2}} \mathrm{~d} \xi_{1} \mathrm{~d} \xi_{2} \\
& \mid \begin{array}{cl}
\cos \varphi\left(V_{, X}\right. & \left.R_{0} \cos \varphi \sin \theta \theta_{, X}\right) \\
\cos \varphi\left(1+U_{, X}\right. & \left.R_{0} \cos \varphi \cos \theta \theta_{, X}\right) \\
\sin \varphi\left(\sin \theta V_{, X}\right. & \left.R_{0} \cos \varphi \theta_{, X}+\cos \theta\left(1+U_{, X}\right)\right)
\end{array} \quad \mathrm{d} \xi_{1} \mathrm{~d} \xi_{2}
\end{aligned}
$$

The virtual displacement field (5) can be recast as:

$$
\mathbf{V}^{*}\left(\mathbf{P}_{0}\right) \quad \mid \begin{array}{ll}
U^{*} & \mathrm{R}_{0} \cos \varphi \cos \theta \theta^{*} \\
V^{*} & \mathrm{R}_{0} \cos \varphi \sin \theta \theta^{*} \\
0 &
\end{array}
$$

From relations (15) and (16), one obtains the contribution of the pressure over the cylindrical surface:

$$
\begin{aligned}
& \int_{\text {Cylindrical surface }} \mathbf{V}^{*}\left(\mathbf{P}_{0}\right) \cdot \mathbf{n} \mathrm{d} S \\
& \quad \pi R_{0}^{2} \int_{0}^{l}\left[U^{*} \sin \theta \theta_{, X} \quad V^{*} \cos \theta \theta_{, X}+\theta^{*}\left(\cos \theta V_{, X} \quad \sin \theta\left(1+U_{, X}\right)\right)\right] \mathrm{d} X
\end{aligned}
$$

The underlined terms stem from the finite rotation adopted in expression (2) for the displacement field.

\subsubsection{Integrals over the ends}

These integrals are computed in the similar way as in the case of dead loads. For instance, from relation (4) and the equality pn $\mathrm{d} S \quad \sigma \cdot \mathbf{n} \mathrm{d} S$ on the end $x \quad l(\boldsymbol{\sigma}$ is the Cauchy stress), we get:

$$
\begin{aligned}
& \int_{\text {End } x=I} \mathbf{V}^{*}\left(\mathbf{P}_{0}\right) \cdot p \mathbf{n} \mathrm{d} S \\
& \quad \int_{\text {End } x=I} \sigma(\mathbf{P}) \cdot \mathbf{n}(\mathbf{P}) \mathrm{d} S+\int_{\text {End } x=I} \mathrm{GP} \times \sigma(\mathbf{P}) \cdot \mathbf{n}(\mathbf{P}) \mathrm{d} S \cdot \mathbf{\Omega}^{*} \quad \mathbf{R V}^{*}+\mathbf{\Gamma} \mathbf{\Omega}^{*}
\end{aligned}
$$


Clearly, the torque $\Gamma$ due to the pressure is zero on the ends. Moreover, by virtue of the assumption that the reference volume $\Omega_{0}$ is a circular cylinder of radius $R_{0}$, the resultant force is $\mathbf{R} p \pi R_{0}^{2} \mathbf{n}$. Hence:

$$
\int_{\text {End } x=\lambda} \mathbf{V}^{*}\left(\mathbf{P}_{0}\right) \cdot p \mathbf{n} \mathrm{d} S \quad p \pi R_{0}^{2}\left(U^{*} \cos \theta+V^{*} \sin \theta\right)
$$

\subsubsection{Virtual work of the pressure}

$$
\begin{array}{rl}
W_{\text {pressure }}^{*} & P \int_{0}^{I_{0}}\left[U^{*} \sin \theta \theta_{, X} \quad V^{*} \cos \theta \theta_{, X}+\theta^{*}\left(\cos \theta V_{, X} \quad \sin \theta\left(1+U_{, X}\right)\right)\right] \mathrm{d} X \\
& +P\left[U^{*} \cos \theta+V^{*} \sin \theta\right]_{0}^{1_{0}}
\end{array}
$$

\subsection{Equilibrium equations and boundary conditions}

Integrating by parts the principle of virtual work (1) and using (10), (12), (14) and (20), lead to the following equilibrium equations for the inflated beam

$$
\begin{aligned}
& \left(N\left(1+U_{, X}\right)\right)_{, X} \quad\left(M \cos \theta \theta_{, X}\right)_{, X}+(T \sin \theta)_{, X} \quad P \sin \theta \theta_{, X} \\
& p_{X} \\
& \left(N V_{, X}\right)_{, X} \quad\left(M \sin \theta \theta_{, X}\right)_{, X} \quad(T \cos \theta)_{, X}+P \cos \theta \theta_{, X} \\
& p_{Y} \\
& \left(M\left(1+U_{, X}\right)\right)_{, X} \cos \theta \quad\left(M V_{, X}\right)_{, X} \sin \theta+\left(\left(1+U_{, X}\right) \cos \theta+V_{, X} \sin \theta\right) T \\
& {\left[\left(\frac{N I_{0}}{S_{0}}+\frac{1}{2} E K \theta_{, X}^{2}+K \gamma^{0}\right) \theta_{, X}\right]_{, X} \quad P\left(\cos \theta V_{, X} \quad \sin \theta\left(1+U_{, X}\right)\right) \quad \mu}
\end{aligned}
$$

together with the boundary conditions:

$$
\begin{aligned}
& N(0)\left(1+U_{, X}(0)\right)+M(0) \cos \theta(0) \theta_{, X}(0) \quad T(0) \sin \theta(0) \quad P \cos \theta(0) \quad X(0) \\
& N\left(I_{0}\right)\left(1+U_{, X}\left(I_{0}\right)\right)+M\left(\lambda_{0}\right) \cos \theta\left(I_{0}\right) \theta_{, X}\left(I_{0}\right)+T\left(I_{0}\right) \sin \theta\left(I_{0}\right) \quad P \cos \theta\left(I_{0}\right) \quad X\left(I_{0}\right) \\
& N(0) V_{, X}(0)+M(0) \sin \theta(0) \theta_{, X}(0)+T(0) \cos \theta(0) \quad P \sin \theta(0) \quad Y(0) \\
& N\left(I_{0}\right) V_{, X}\left(I_{0}\right)+M\left(\lambda_{0}\right) \sin \theta\left(I_{0}\right) \theta_{, X}\left(I_{0}\right)+T\left(I_{0}\right) \cos \theta\left(I_{0}\right) \quad P \sin \theta\left(I_{0}\right) \quad Y\left(I_{0}\right) \\
& M(0)\left(1+U_{, X}(0)\right) \cos \theta(0)+M(0) \sin \theta(0) V_{, X}(0) \\
& +\left(\frac{N(0) I_{0}}{S_{0}}+\frac{1}{2} E K \theta_{, X}^{2}(0)+K \gamma^{0}\right) \theta_{, X}(0) \quad \Gamma(0)
\end{aligned}
$$




$$
\begin{gathered}
M\left(I_{0}\right)\left(1+U_{, X}\left(I_{0}\right)\right) \cos \theta\left(I_{0}\right)+M\left(I_{0}\right) \sin \theta\left(I_{0}\right) V_{, X}\left(I_{0}\right) \\
+\left(\frac{N\left(I_{0}\right) I_{0}}{S_{0}}+\frac{1}{2} E K \theta_{, X}^{2}\left(I_{0}\right)+K \gamma^{0}\right) \theta_{, X}\left(I_{0}\right) \quad \Gamma\left(I_{0}\right)
\end{gathered}
$$

It remains to substitute relations (9) for $(N, M, T)$ in Eq. (21) to obtain a set of three nonlinear equations with three unknowns $(U, V, \theta)$. Relations (21) (22f) are similar to those obtained by Fichter [2], the additional terms are due to the fact that here the displacement field (2) involves finite rotation.

\section{Linearized equations}

In this work, we shall confine ourselves to small deformations so as to deal with linearized equations which are simpler to solve. The linearization of the equations will be performed about the reference configuration which is in a pre-stressed state. For instance, in the case of bending, the pre-stress is due to the preliminary inflation of the beam; in the case of buckling, the pre-stress also includes the compressive load.

The linearization process is based on usual hypotheses on the magnitudes of the displacements and the rotation:

(i) $\mathrm{V} / l_{0}$ and $\theta$ are infinitesimal quantities of order 1 ,

(ii) $\mathrm{U} / l_{0}$ is infinitesimal of order 2.

Furthermore, the following assumptions are made on the initial stresses:

(i) The initial axial stress $\Sigma_{X X}^{0}$ is constant over the cross-section. Thus, $M^{0} \gamma^{0} \quad 0$.

(ii) The initial shear stress $\Sigma_{X Y}^{0}$ is zero. Thus, $T^{0} \quad 0$.

Taking these assumptions into account, one arrives at the linearized expressions for the constitutive laws (9)

$$
N \quad N^{0} \quad T \quad k G S_{0}\left(\begin{array}{llll}
V_{, x} & \theta) & M & E I_{0} \theta_{, X}
\end{array}\right.
$$

From (21), the linearized equilibrium equations are:

$$
\begin{aligned}
& N_{, X}^{0} \quad p_{X} \\
& \left(N^{0}+k G S_{0}\right) V_{, X^{2}}+\left(P+k G S_{0}\right) \theta_{, X} \quad p_{Y} \\
& \quad\left(E+\frac{N^{0}}{S_{0}}\right) I_{0} \theta_{, X^{2}}+\left(P+k G S_{0}\right)\left(V_{, X} \quad \theta\right) \quad \mu
\end{aligned}
$$

The linearized boundary conditions are derived from (22a) (22f):

$$
\begin{array}{llr}
N^{0}(0) & P & X(0) \\
N^{0}\left(l_{0}\right) & P & X\left(l_{0}\right)
\end{array}
$$




$$
\begin{array}{lll}
\left(N^{0}(0)+k G S_{0}\right) V_{, X}(0) & \left(P+k G S_{0}\right) \theta(0) & Y(0) \\
\left(N^{0}\left(l_{0}\right)+k G S_{0}\right) V_{, X}\left(l_{0}\right) & \left(P+k G S_{0}\right) \theta\left(l_{0}\right) & Y\left(l_{0}\right) \\
\left(E+\frac{N^{0}(0)}{S_{0}}\right) I_{0} \theta_{, X}(0) & \Gamma(0) \\
\left(E+\frac{N^{0}\left(I_{0}\right)}{S_{0}}\right) I_{0} \theta_{, X}\left(I_{0}\right) & \Gamma\left(I_{0}\right)
\end{array}
$$

The linearized Eqs. (24) (25f) are quite similar to those obtained by Fichter [2]. Yet there are two slight differences: (i) the linearization process, based on a well-defined reference configuration, definitely shows that the obtained equations form a set of linearized equations in terms of displacements $U, V$ and rotation $\theta$; (ii) the pressure is equally involved in the equilibrium equations and boundary conditions, so that it modifies both the Young modulus and the shear modulus. This modification will be more explicit in the bending and buckling examples considered in the next sections.

In addition, it should be mentioned that all the reference dimensions (e.g. length $l_{0}$, cross-section area $S_{0}$ and second moment of area $I_{0}$ ) are themselves functions of the pressure $p$. Computing their values corresponding to a given pressure may be difficult, but this is an independent subject which actually is common to every problem with a prestressed reference configuration.

\subsection{Solution scheme}

Eq. (24) and the boundary conditions (25a) and (25b), related to the axial displacement $U$, are decoupled from the rest and can easily be integrated. Let us describe how to solve Eq. (24) and the boundary conditions (25c) (25f), related to the deflection $V$ and the rotation $\theta$.

Eq. (24) can be used to eliminate $V_{, X}$ as

$$
V_{, X} \quad \frac{\left(P+k G S_{0}\right)}{\left(N^{0}+k G S_{0}\right)} \theta \quad \frac{C}{\left(N^{0}+k G S_{0}\right)}
$$

where $C \int p_{Y} \mathrm{~d} X$ denotes a primitive of the load $p_{Y}$. Inserting (26) into (24) provides a differential equation of second order in $\theta$ :

$$
\left(E+\frac{N^{0}}{S_{0}}\right) I_{0} \theta_{, X^{2}}+\frac{\left(P+k G S_{0}\right)}{\left(N^{0}+k G S_{0}\right)}\left(P \quad N^{0}\right) \theta \quad \mu+C \frac{\left(P+k G S_{0}\right)}{\left(N^{0}+k G S_{0}\right)}
$$

The above equation is similar to that obtained in [5]. Integrating it leads to three constants of integration, whereas integrating (26) leads to one additional constant. These four constants are determined by means of the boundary conditions (25c) (25f). 


\section{Bending of an inflatable beam}

In this section, we consider, as the first example, the linearized problem of an inflated cantilever beam under bending. The beam is made of a cylindrical membrane, its reference length is $l_{0}$, its reference radius $R_{0}$ and its reference thickness $h_{0}$. The beam is built-in at end $X \quad 0$, subjected to an internal pressure $\mathrm{p}$ and a transverse force $F \mathbf{y}$ at end $X \quad l_{0}$.

\subsection{Deflection and rotation}

From the equilibrium Eq. (24) and the boundary conditions (25a) and (25b), one readily gets the initial axial force $N^{0}(X) \quad P$. Hence, Eqs. (26) and (27) become:

$$
\begin{aligned}
& V_{, X} \quad \theta \quad \frac{C}{P+k G S_{0}} \quad \text { (Here, } C \text { is a constant of integration) } \\
& \left(E+\frac{P}{S_{0}}\right) I_{0} \theta_{, X^{2}} \quad C
\end{aligned}
$$

Taking into account the boundary conditions (25c) (25f)

$$
\begin{aligned}
& V(0) \quad \theta(0) \quad \theta_{, X}\left(l_{0}\right) \quad 0 \\
& \left(P+k G S_{0}\right)\left(V_{, X}\left(l_{0}\right) \quad \theta\left(l_{0}\right)\right) \quad F
\end{aligned}
$$

one obtains the deflection and the rotation along the beam:

$$
\begin{aligned}
& V(x) \frac{F}{\left(E+P / S_{0}\right) I_{0}}\left(\frac{I_{0} x^{2}}{2} \frac{x^{3}}{6}\right)+\frac{F x}{P+k G S_{0}} \\
& \theta(x) \frac{F}{\left(E+P / S_{0}\right) I_{0}}\left(I_{0} x \frac{x^{2}}{2}\right)
\end{aligned}
$$

The solution is linear with respect to force $F$, yet non-linear with respect to the pressure. First, the pressure appears in the denominators of the right-hand sides of (32) and (33). Second, the reference dimensions $l_{0}, S_{0}$, and $I_{0}$ themselves depend on the pressure; however, further numerical results show that this dependence may not be too strong.

If the internal pressure is zero, these relations give the well-known results for the Timoshenko beam model. However, contrary to a classical beam, here the inflatable beam is made of a membrane, so the pressure cannot be equal to zero for the beam not to collapse. This fact will be discussed below in connection with the validity of the solution. The influence of the internal pressure on the beam response is clearly shown in the previous relations: the inflation amounts to strengthen the Young modulus and the shear modulus. In particular, when $p$ tends to infinity, so do the equivalent material properties and the deflection and the rotation are identically zero.

In a recent paper [5], a solution for the bending problem was obtained by using the force formulation. The Taylor expansion of the deflection expression therein with respect to 
the resultant pressure $P$ shows that the first non-zero term in $P$ is a quadratic term:

$$
V(x) \quad \frac{F}{E I_{0}}\left(1 \quad \frac{P^{2}}{\left(k G S_{0}\right)^{2}}\right)\left(\begin{array}{ll}
\frac{I_{0} x^{2}}{2} & \frac{x^{3}}{6}
\end{array}\right)+\frac{F x}{k G S_{0}}
$$

On the other hand, the Taylor expansion of relation (32) shows that the first non-zero terms in $P$ are linear:

$$
V(x) \quad \frac{F}{E I_{0}}\left(\begin{array}{ll}
1 & \frac{P}{E S_{0}}
\end{array}\right)\left(\begin{array}{ll}
\frac{I_{0} x^{2}}{2} & \frac{x^{3}}{6}
\end{array}\right)+\frac{F x}{k G S_{0}}\left(\begin{array}{ll}
1 & \frac{P}{k G S_{0}}
\end{array}\right)
$$

Relations (34) and (35) show that whichever of the formulations is used to estimate the deflection, one obtains the same leading terms which come from the beam and yarn compliances. Not surprisingly, the additional terms are different due to the very differences between the force and displacement formulations.

Eventually, it should be mentioned that the deflection of inflated panels obtained in [4] is identical to (32), provided the Young modulus $E$ in [4] is replaced by $E+P / S_{0}$. Also, studies in progress show that the finite element derived in [6] for inflatable panels is identical to that for inflatable beams, when the same substitution is carried out.

\subsection{Limit of validity of the solution}

Of course, one has to check a posteriori that the deflection and the rotation given by (32) and (33) satisfy the small deformation hypothesis required by the linearization process. Yet there is another condition for the solution to hold: as mentioned above, since the inflatable beam is made of a membrane, the internal pressure must be high enough. More precisely, the solution is valid if the principal stresses at any point in the beam are nonnegative. One can check that this amounts to saying that the axial stress $\Sigma_{X X}$ at the point $\left(\begin{array}{llll}X & 0, Y & R_{0}, Z & 0\end{array}\right)$ is non-negative:

$$
\Sigma_{X X} \quad \Sigma_{X X}^{0} \quad \frac{M}{I_{0}} Y \quad \frac{p R_{0}}{2 h_{0}} \quad \frac{F I_{0}}{\pi R_{0}^{3} h_{0}} R_{0} \geq 0 \quad \leftrightarrow \quad F \leq \frac{\pi R_{0}^{3} p}{2 I_{0}}
$$

Recall that the reference length $l_{0}$ and radius $R_{0}$ are (increasing) functions of the pressure $p$. Inequality (36) shows that given a force $F$, the internal pressure must be high enough for the bending solution to be meaningful. There exists a lower bound for the pressure, referred to as the wrinkling pressure of the beam, below which a wrinkle appears first at the point ( $X \quad 0, Y \quad R_{0}, Z \quad 0$ ) and the bending solution is no longer valid.

\subsection{Comparison with $3 D$ membrane finite element results}

In order to assess the proposed theory, the numerical values of the deflection (32) will be compared to three-dimensional membrane finite element results. The membrane computations are carried out using a general purpose non-linear finite element program, based on the total Lagrangian formulation. The beam is modelled as a three-dimensional membrane structure; the membrane elements have zero bending stiffness and satisfy the usual plane stress condition. The three-dimensional constitutive law is the Saint-Venant 
Kirchhoff one, characterized by the Young modulus $E$ and Poisson ratio $\nu$, or equivalently, by the Lamé constants $(\lambda, \mu)$.

Discretizing the virtual power principle by the finite element technique leads to a nonlinear equilibrium equation in terms of the displacement field, which is solved using the Newton iterative scheme. The total tangent stiffness matrix is the sum of (i) the stiffness due to the internal forces and (ii) the stiffness due to the pressure. As the matter is standard, let us just make a brief reminder for the internal forces. By denoting NNE the node number of an element, the element stiffness matrix due to the internal force writes

$$
\forall i, j \in[1,3 \mathrm{NNE}], K_{i j}^{e} \quad \int_{\text {element }} N_{a, \alpha} N_{b, \beta}\left(\delta_{p q} \Sigma^{\beta \alpha}+x_{p, \gamma} x_{q, \delta} \frac{\partial \Sigma^{\alpha \gamma}}{\partial E_{\beta \delta}}\right) h_{0} \mathrm{~d} S_{0}
$$

where $\mathbf{x}$ denotes the current position of a particle belonging to the reference middle surface $S_{0}$ of the membrane; $N_{a}, a \in[1, \mathrm{NNE}]$, are the shape functions used to define the geometry as well as the displacement in each element (isoparametric element). All the derivations are performed with respect to curvilinear coordinates defining the membrane element. Here, integers $a, b \in[1, \mathrm{NNE}]$ and $p, q \in\{1,2,3\}$ are related to indices $i$ and $j$ by $i \quad 3(a$ $1)+p, j \quad 3(b \quad 1)+q$. Implicit summation is carried out over $\alpha, \beta, \gamma, \delta, \in\{1,2\}$.

Taking into account the plane stress condition $\Sigma^{33} \quad 0$, one readily gets the expression for the tangent modulus

$$
\frac{\partial \Sigma^{\alpha \beta}}{\partial E_{\delta \gamma}} \quad \mu\left(G^{\alpha \gamma} G^{\beta \delta}+G^{\alpha \delta} G^{\beta \gamma}\right)+\frac{2 \lambda \mu}{2 \mu+\lambda} G^{\alpha \beta} G^{\gamma \delta}
$$

where $G^{\alpha \beta}$ the contravariant components of the metric tensor defined on the reference middle surface.

Also, the path-following and branch switching techniques are included in the numerical scheme, in order to deal with possible limit and bifurcation points, as seen in the buckling case below. For membrane structures, an artificial initial stress has to be added in the first increment. It is, however, removed in the next increments.

It should be emphasized that in the $3 \mathrm{D}$ membrane finite element solution, the loading is applied in two successive stages: first, the beam is inflated to a given pressure $p$, and then a force $F$ is applied. At the very beginning of the first stage, the internal pressure is zero and the beam is in a natural (or stress free) state. On the other hand, the reference configuration, which corresponds to the beginning of the second stage (before the force $F$ is applied), is in a pre-stressed state. To clearly distinguish between the two states, we use index $\varnothing$ to denote the quantities in the natural state, as opposed to the usual index o for quantities related to the reference configuration. Thus, $l_{\varnothing}, R_{\varnothing}$ and $h_{\varnothing}$ designate the natural length, radius and thickness, respectively; while $l_{0}, R_{0}$ and $h_{0}$ the reference dimensions, which vary as functions of the pressure.

\subsection{Numerical results}

The numerical computations are performed with three values of natural radius $R_{\varnothing}$, three values of natural length $l_{\varnothing}$ and four values of pressure $p$, while other quantities 
Table 1

Data set for numerical computations

\begin{tabular}{|c|c|c|c|c|}
\hline \multirow{2}{*}{\multicolumn{2}{|c|}{$\begin{array}{l}\text { Natural thickness, } h_{\varnothing}(\mathrm{m}) \\
\text { Young modulus, } E\left(\mathrm{~N} / \mathrm{m}^{2}\right)\end{array}$}} & \multicolumn{2}{|c|}{$125 \times 10^{-6}$} & \\
\hline & & \multicolumn{2}{|c|}{$2.5 \times 10^{9}$} & \\
\hline Poisson ratio, $\nu$ & & \multicolumn{2}{|c|}{0.3} & \\
\hline Correction shear coefficient, $k$ & & & & \\
\hline Natural radius, $R_{\varnothing}(\mathrm{m})$ & 0.04 & 0.06 & 0.08 & \\
\hline Natural length, $l_{\varnothing}(\mathrm{m})$ & 0.65 & 0.90 & 1.15 & \\
\hline Pressure, $p\left(\mathrm{~N} / \mathrm{m}^{2}\right)$ & $0.5 \times 10^{5}$ & $10^{5}$ & $1.5 \times 10^{5}$ & $2 \times 10^{5}$ \\
\hline
\end{tabular}

(the natural thickness $h_{\varnothing}$, Young modulus $E$, Poisson ratio $\nu$ and correction shear factor $k$ ) remain fixed, as shown in Table 1.

As mentioned above, the correction shear coefficient $k$, introduced after expression (9) for the shear force $T$, is taken equal to the usual value for circular thin tubes, i.e. $k \quad 0.5$.

As the problem is linear with respect to force $F$, we take $F \quad 1 \mathrm{~N}$ in all the numerical computations. Fig. 2 shows a typical mesh used in $3 \mathrm{D}$ membrane finite element computations, containing 2401 nodes and 768 eight-node quadrilaterals and six-node triangles. The reference length $l_{0}$, radius $R_{0}$ and thickness $h_{0}$ are computed as functions of the internal pressure by using the well-known elastic small strain analytical solution for thin tubes. For instance, the expression for the reference thickness is:

$$
h_{0} \quad h_{\varnothing} \quad \frac{3 \nu}{E} \frac{p R_{\varnothing}}{2}
$$

With the chosen natural radii and pressures, it is found that the reference thickness is virtually identical to the natural thickness, to within $2 \%$ at most. In the case of more complex geometries, one should use a membrane finite element solution for obtaining the reference length $l_{0}$ and radius $R_{0}$, and an appropriate estimate for the reference thickness $h_{0}$.

Table 2 and Fig. 3 give the maximum deflection in the cantilever given by (32) as a function of the length of the beam and the internal pressure, in comparison with the deflection obtained by 3D membrane finite elements. Given one fixed natural radius $R_{\varnothing}$, one natural length $l_{\varnothing}$, there are in Table 2 four reference lengths $l_{0}$, depending on whether pressure $p$ is $0.5 \times 10^{5}, 10^{5}, 1.5 \times 10^{5}$ or $2 \times 10^{5}$ pa, respectively.

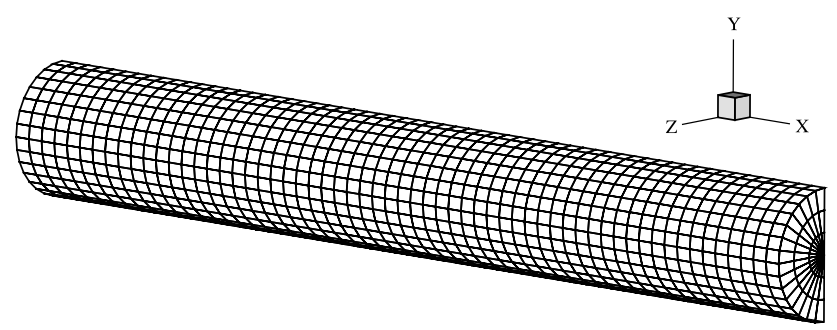

Fig. 2. A typical mesh used in 3D membrane finite element computations. 
Table 2

Maximum deflection in the cantilever given by the inflated beam theory, compared with the 3D finite element results. All deflections are obtained with force $\mathrm{F}=1 \mathrm{~N}$.

\begin{tabular}{|c|c|c|c|c|c|c|c|}
\hline \multirow{2}{*}{$\begin{array}{l}\text { Natural } \\
\text { length, } \\
l_{\varnothing}(\mathrm{m})\end{array}$} & \multirow{2}{*}{$\begin{array}{l}\text { Pressure } \\
\left(\mathrm{N} / \mathrm{m}^{2}\right)\end{array}$} & \multirow{2}{*}{$\begin{array}{l}\text { Reference } \\
\text { length, } \\
l_{0}(\mathrm{~m})\end{array}$} & \multirow{2}{*}{$\begin{array}{l}\text { Reference } \\
\text { radius, } \\
R_{0}(\mathrm{~m})\end{array}$} & \multirow{2}{*}{$\begin{array}{l}\text { Reference } \\
\text { thickness, } \\
h_{0}(\mathrm{~m})\end{array}$} & \multicolumn{3}{|l|}{ Deflection (m) } \\
\hline & & & & & $\begin{array}{l}\text { By the inflat- } \\
\text { able beam } \\
\text { theory }\end{array}$ & $\begin{array}{l}\text { By the mem- } \\
\text { brane finite } \\
\text { element }\end{array}$ & $\begin{array}{l}\text { Diff- } \\
\text { eren- } \\
\text { ce } \\
(\%)\end{array}$ \\
\hline \multicolumn{8}{|c|}{ Case 1. Natural radius $R_{\varnothing}=0.04 \mathrm{~m}$} \\
\hline \multirow[t]{4}{*}{0.65} & $5.0 \times 10^{4}$ & $6.508 \times 10^{-1}$ & $4.022 \times 10^{-2}$ & $1.246 \times 10^{-4}$ & $1.481 \times 10^{-3}$ & $1.487 \times 10^{-3}$ & -0.4 \\
\hline & $1.0 \times 10^{5}$ & $\begin{array}{l}6.517 \\
\times 10^{-1} \times 10^{-01}\end{array}$ & $\begin{array}{l}4.044 \times \\
10^{-2} \times 10^{-02}\end{array}$ & $1.243 \times 10^{-4}$ & $1.462 \times 10^{-3}$ & $1.461 \times 10^{-3}$ & 0.1 \\
\hline & $1.5 \times 10^{5}$ & $6.525 \times 10^{-1}$ & $4.065 \times 10^{-2}$ & $1.239 \times 10^{-4}$ & $1.443 \times 10^{-3}$ & $1.436 \times 10^{-3}$ & 0.5 \\
\hline & $2.0 \times 10^{5}$ & $6.533 \times 10^{-1}$ & $4.087 \times 10^{-2}$ & $1.236 \times 10^{-4}$ & $1.425 \times 10^{-3}$ & $1.413 \times 10^{-3}$ & 0.9 \\
\hline \multirow[t]{4}{*}{0.9} & $5.0 \times 10^{4}$ & $9.012 \times 10^{-1}$ & $4.022 \times 10^{-2}$ & $1.246 \times 10^{-4}$ & $3.877 \times 10^{-3}$ & $3.886 \times 10^{-3}$ & -0.2 \\
\hline & $1.0 \times 10^{5}$ & $9.023 \times 10^{-1}$ & $4.044 \times 10^{-2}$ & $1.243 \times 10^{-4}$ & $3.828 \times 10^{-3}$ & $3.820 \times 10^{-3}$ & 0.2 \\
\hline & $1.5 \times 10^{5}$ & $9.035 \times 10^{-1}$ & $4.065 \times 10^{-2}$ & $1.239 \times 10^{-4}$ & $3.779 \times 10^{-3}$ & $3.757 \times 10^{-3}$ & 0.6 \\
\hline & $2.0 \times 10^{5}$ & $9.046 \times 10^{-1}$ & $4.087 \times 10^{-2}$ & $1.236 \times 10^{-4}$ & $3.731 \times 10^{-3}$ & $3.696 \times 10^{-3}$ & 1.0 \\
\hline \multirow[t]{4}{*}{1.15} & $5.0 \times 10^{4}$ & 1.151 & $4.022 \times 10^{-2}$ & $1.246 \times 10^{-4}$ & $8.041 \times 10^{-3}$ & $8.054 \times 10^{-3}$ & -0.2 \\
\hline & $1.0 \times 10^{5}$ & 1.153 & $4.044 \times 10^{-2}$ & $1.243 \times 10^{-4}$ & $7.939 \times 10^{-3}$ & $7.920 \times 10^{-3}$ & 0.2 \\
\hline & $1.5 \times 10^{5}$ & 1.154 & $4.065 \times 10^{-2}$ & $1.239 \times 10^{-4}$ & $7.838 \times 10^{-3}$ & $7.791 \times 10^{-3}$ & 0.6 \\
\hline & $2.0 \times 10^{5}$ & 1.156 & $4.087 \times 10^{-2}$ & $1.236 \times 10^{-4}$ & $7.740 \times 10^{-3}$ & $7.665 \times 10^{-3}$ & 1.0 \\
\hline \multicolumn{8}{|c|}{ Case 2 Natural radius $R_{\varnothing}=0.06 \mathrm{~m}$} \\
\hline \multirow[t]{4}{*}{0.65} & $5.0 \times 10^{4}$ & $6.512 \times 10^{-1}$ & $6.049 \times 10^{-2}$ & $1.245 \times 10^{-4}$ & $4.514 \times 10^{-4}$ & $4.554 \times 10^{-4}$ & -0.9 \\
\hline & $1.0 \times 10^{5}$ & $6.525 \times 10^{-1}$ & $6.098 \times 10^{-2}$ & $1.239 \times 10^{-4}$ & $4.427 \times 10^{-4}$ & $4.426 \times 10^{-4}$ & 0.0 \\
\hline & $1.5 \times 10^{5}$ & $6.537 \times 10^{-1}$ & $6.147 \times 10^{-2}$ & $1.234 \times 10^{-4}$ & $4.342 \times 10^{-4}$ & $4.313 \times 10^{-4}$ & 0.7 \\
\hline & $2.0 \times 10^{5}$ & $6.550 \times 10^{-1}$ & $6.196 \times 10^{-2}$ & $1.228 \times 10^{-4}$ & $4.259 \times 10^{-4}$ & $4.207 \times 10^{-4}$ & 1.2 \\
\hline \multirow[t]{4}{*}{0.9} & $5.0 \times 10^{4}$ & $9.017 \times 10^{-1}$ & $6.049 \times 10^{-2}$ & $1.245 \times 10^{-4}$ & $1.163 \times 10^{-3}$ & $1.166 \times 10^{-3}$ & -0.3 \\
\hline & $1.0 \times 10^{5}$ & $9.035 \times 10^{-1}$ & $6.098 \times 10^{-2}$ & $1.239 \times 10^{-4}$ & $1.141 \times 10^{-3}$ & $1.136 \times 10^{-3}$ & 0.4 \\
\hline & $1.5 \times 10^{5}$ & $9.052 \times 10^{-1}$ & $6.147 \times 10^{-2}$ & $1.234 \times 10^{-4}$ & $1.119 \times 10^{-3}$ & $1.108 \times 10^{-3}$ & 1.0 \\
\hline & $2.0 \times 10^{5}$ & $9.069 \times 10^{-1}$ & $6.196 \times 10^{-2}$ & $1.228 \times 10^{-4}$ & $1.098 \times 10^{-3}$ & $1.081 \times 10^{-3}$ & 1.5 \\
\hline \multirow[t]{4}{*}{1.15} & $5.0 \times 10^{4}$ & 1.152 & $6.049 \times 10^{-2}$ & $1.245 \times 10^{-4}$ & $2.395 \times 10^{-3}$ & $2.396 \times 10^{-3}$ & -0.1 \\
\hline & $1.0 \times 10^{5}$ & 1.154 & $6.098 \times 10^{-2}$ & $1.239 \times 10^{-4}$ & $2.349 \times 10^{-3}$ & $2.336 \times 10^{-3}$ & 0.6 \\
\hline & $1.5 \times 10^{5}$ & 1.157 & $6.147 \times 10^{-2}$ & $1.234 \times 10^{-4}$ & $2.305 \times 10^{-3}$ & $2.279 \times 10^{-3}$ & 1.1 \\
\hline & $2.0 \times 10^{5}$ & 1.159 & $6.196 \times 10^{-2}$ & $1.228 \times 10^{-4}$ & $2.261 \times 10^{-3}$ & $2.225 \times 10^{-3}$ & 1.6 \\
\hline \multicolumn{8}{|c|}{ Case 3. Natural radius $R_{\varnothing}=0.08 \mathrm{~m}$} \\
\hline \multirow[t]{4}{*}{0.65} & $5.0 \times 10^{4}$ & $6.517 \times 10^{-1}$ & $8.087 \times 10^{-2}$ & $1.243 \times 10^{-4}$ & $1.983 \times 10^{-4}$ & $2.016 \times 10^{-4}$ & -1.7 \\
\hline & $1.0 \times 10^{5}$ & $6.533 \times 10^{-1}$ & $8.174 \times 10^{-2}$ & $1.236 \times 10^{-4}$ & $1.931 \times 10^{-4}$ & $1.939 \times 10^{-4}$ & -0.4 \\
\hline & $1.5 \times 10^{5}$ & $6.550 \times 10^{-1}$ & $8.261 \times 10^{-2}$ & $1.228 \times 10^{-4}$ & $1.881 \times 10^{-4}$ & $1.873 \times 10^{-4}$ & 0.5 \\
\hline & $2.0 \times 10^{5}$ & $6.567 \times 10^{-1}$ & $8.348 \times 10^{-2}$ & $1.221 \times 10^{-4}$ & $1.833 \times 10^{-4}$ & $1.811 \times 10^{-4}$ & 1.2 \\
\hline \multirow[t]{4}{*}{0.9} & $5.0 \times 10^{4}$ & $9.023 \times 10^{-1}$ & $8.087 \times 10^{-2}$ & $1.243 \times 10^{-4}$ & $5.000 \times 10^{-4}$ & $5.021 \times 10^{-4}$ & -0.4 \\
\hline & $1.0 \times 10^{5}$ & $9.046 \times 10^{-1}$ & $8.174 \times 10^{-2}$ & $1.236 \times 10^{-4}$ & $4.872 \times 10^{-4}$ & $4.847 \times 10^{-4}$ & 0.5 \\
\hline & $1.5 \times 10^{5}$ & $9.069 \times 10^{-1}$ & $8.261 \times 10^{-2}$ & $1.228 \times 10^{-4}$ & $4.749 \times 10^{-4}$ & $4.688 \times 10^{-4}$ & 1.3 \\
\hline & $2.0 \times 10^{5}$ & $9.092 \times 10^{-1}$ & $8.348 \times 10^{-2}$ & $1.221 \times 10^{-4}$ & $4.629 \times 10^{-4}$ & $4.540 \times 10^{-4}$ & 2.0 \\
\hline \multirow[t]{4}{*}{1.15} & $5.0 \times 10^{4}$ & 1.153 & $8.087 \times 10^{-2}$ & $1.243 \times 10^{-4}$ & $1.020 \times 10^{-3}$ & $1.020 \times 10^{-3}$ & 0.0 \\
\hline & $1.0 \times 10^{5}$ & 1.156 & $8.174 \times 10^{-2}$ & $1.236 \times 10^{-4}$ & $9.940 \times 10^{-4}$ & $9.861 \times 10^{-4}$ & 0.8 \\
\hline & $1.5 \times 10^{5}$ & 1.159 & $8.261 \times 10^{-2}$ & $1.228 \times 10^{-4}$ & $9.690 \times 10^{-4}$ & $9.545 \times 10^{-4}$ & 1.5 \\
\hline & $2.0 \times 10^{5}$ & 1.162 & $8.348 \times 10^{-2}$ & $1.221 \times 10^{-4}$ & $9.448 \times 10^{-4}$ & $9.247 \times 10^{-4}$ & 2.2 \\
\hline
\end{tabular}


As shown in Table 2, the values for the deflection obtained by the inflatable beam theory are in good agreement with that obtained with the membrane finite element. Over the whole range of the computation, the differences are lower than $2.2 \%$.

As expected, the maximum deflection $\mathrm{v}$ increases along with the tube length, and it decreases as the internal pressure increases. With $R_{\varnothing} 0.08 \mathrm{~m}$, for instance, $\mathrm{v}$ is multiplied by about five from $l_{\varnothing} \quad 0.65$ to $1.15 \mathrm{~m}$. With $R_{\varnothing} \quad 0.08 \mathrm{~m}$ and $l_{\varnothing} \quad 1.15 \mathrm{~m}, v$ decreases by about $9 \%$ when the pressure varies from $0.5 \times 10^{5}$ to $2 \times 10^{5}$.

Relation (36) is a rather intricate non-linear inequality with the wrinkling pressure $p$ as unknown. A satisfactory approximation can be obtained by making in this inequality $l_{0} \approx l_{\varnothing}$ and $R_{0} \approx R_{\varnothing}$, which leads to the following simple bound where the right-hand side is known:

$$
p \geq \frac{2 F \lambda_{\varnothing}}{\pi R_{\varnothing}^{3}}
$$

The wrinkling pressure given by (40) is shown in Table 3, it is also represented in Fig. 3 in dashed lines. For each natural radius $R_{\varnothing}$, one finds three wrinkling pressures corresponding to three natural length $l_{\varnothing}$.

Eventually, note that the pressure plays a crucial role in the theory: if the term $P$ were discarded in relation (32), the discrepancy between the beam theory and the membrane finite element computation should reach $5.4 \%$.

Concerning the role of the correction coefficient $k$, other numerical computations show that if $k$ is given very large values so as to cancel the shear effect and switch from the Timoshenko to the Euler Bernoulli model then the maximum difference between the beam theory and the 3D membrane finite element computation reaches $12 \%$.

\section{Buckling of an inflatable beam}

Consider now the same cantilever beam as in the previous section, and replace the bending force with an axial compressive force. As in the case of a classical beam, experiments show that, given an internal pressure, for low values of the compressive force $F$ there exists a unique solution corresponding to a uniaxial stress state where the beam remains straight. On the other hand, when force $F$ reaches some critical values, non-trivial solutions are possible, which correspond to a bent position of the beam. Let us compute such critical values by means of the linearized equilibrium Eq. (24).

\subsection{Buckling force}

Here, the equilibrium Eq. (24) and the boundary condition (25a) and (25b) directly yield the initial axial force $N^{0}(X) \quad P \quad F$. Hence, Eqs. (26) (27) become:

$$
V_{, X} \quad \frac{P+k G S_{0}}{P \quad F+k G S_{0}} \theta \quad \frac{C}{P \quad F+k G S_{0}} \quad(C \text { is a constant of integration })
$$



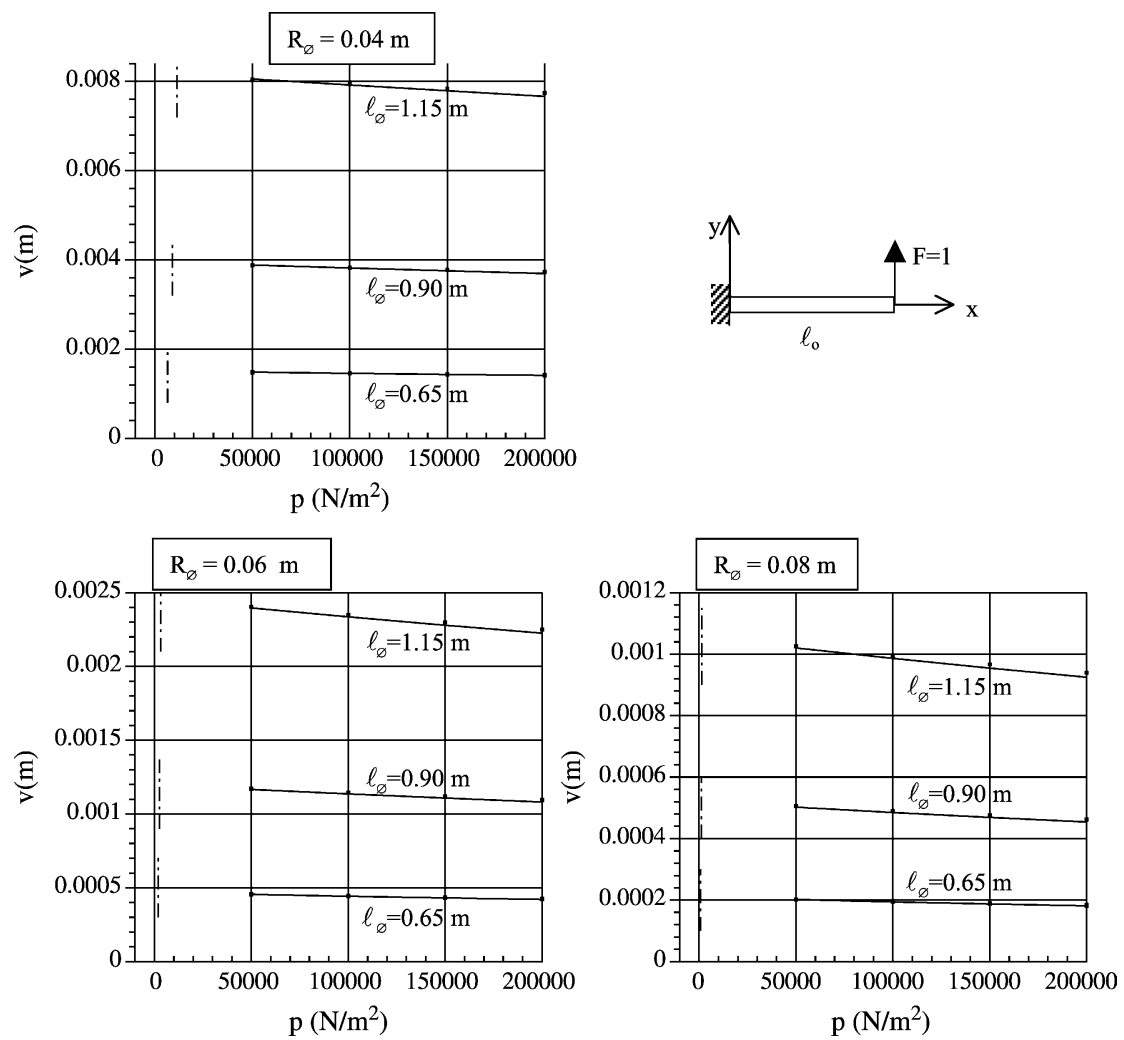

Fig. 3. Maximum deflection in the cantilever. (•) Inflatable beam theory, ( ) membrane finite element, ( • • ) wrinkling pressure.

$$
\left(E+\frac{P \quad F}{S_{0}}\right) I_{0} \theta_{, X^{2}}+\frac{\left(P+k G S_{0}\right) F}{P \quad F+k G S_{0}} \theta \quad \frac{P+k G S_{0}}{P \quad F+k G S_{0}} C
$$

The boundary conditions $(25 \mathrm{c})(25 \mathrm{f})$ write:

$$
\begin{aligned}
& V(0) \quad \theta(0) \quad \theta_{, X}\left(l_{0}\right) \quad 0 \\
& \left(\begin{array}{llll}
P & \left.F+k G S_{0}\right) V_{X X}\left(l_{0}\right) & \left.\left(P+k G S_{0}\right) \theta\left(l_{0}\right)\right) & 0
\end{array}\right.
\end{aligned}
$$

Relation (44) gives $C \quad 0$, whence the differential equation governing the rotation $\theta$ about the $z$-axis

$$
\theta_{, x x}+\Omega^{2} \theta \quad 0
$$

where

$$
\Omega^{2} \frac{F\left(P+k G S_{0}\right)}{(E+(P \quad F) / S) I_{0}\left(P \quad F+k G S_{0}\right)}
$$


Table 3

Wrinkling pressure $\left(\mathrm{N} / \mathrm{m}^{2}\right)$ given by relation (36)

\begin{tabular}{lllr}
\hline Natural radius $R_{\varnothing}(\mathrm{m})$ & \multicolumn{2}{l}{ Natural length $l_{\varnothing}(\mathrm{m})$} & 1.15 \\
\cline { 2 - 4 } & 0.65 & 0.90 & 11,439 \\
0.04 & 6466 & 8952 & 3389 \\
0.06 & 1916 & 2653 & 1430 \\
0.08 & 808 & 1119 & 14 \\
\hline
\end{tabular}

It can be checked in usual numerical applications that quantity $\Omega^{2}$ is positive indeed. The boundary conditions on the rotation (43) entail the following relations similar to those obtained in the classical Timoshenko beam theory:

$$
\begin{aligned}
& \theta \quad B \sin \Omega X \text { and } V \quad \frac{P+k G S_{0}}{P \quad F+k G S_{0}} \frac{B}{\Omega}(1 \quad \cos \Omega X) \text {, } \\
& \Omega l_{0} \quad(2 n \quad 1) \pi / 2
\end{aligned}
$$

Coefficient $B$ remains undetermined and $\mathrm{n}$ is an integer, which is taken equal to 1 in the sequel since we are concerned with the fundamental buckling mode only. From relation (46), the critical force $F_{\mathrm{c}}$ is obtained as the smallest root of the quadratic equation:

$$
\begin{aligned}
& F^{2} \frac{\Omega^{2} I_{0}}{S_{0}} \quad F\left[\Omega^{2}(E+P / S) I_{0}+\left(P+k G S_{0}\right)\left(1+\Omega^{2} I_{0} / S_{0}\right)\right] \\
& \quad+\Omega^{2}(E+P / S) I_{0}\left(P+k G S_{0}\right) \quad 0
\end{aligned}
$$

For usual numerical values, it is found that Eq. (48) gives one finite root to be adopted and one virtually infinite to be discarded. Clearly, as the inflation pressure increases both the Young modulus and the shear modulus, it also raises the buckling force.

\subsection{Limit of validity of the solution}

Like the bending case, the buckling force given by (48) is meaningful only if the internal pressure is high enough. Before buckling takes place, the principal axes of stress at every point are directed along the cylindrical base vectors, so that the validity condition of the solution writes

$$
\Sigma_{X X}^{0}>0 \leftrightarrow N^{0} \quad P \quad F>0 \leftrightarrow F<P \quad p \cdot \pi R_{0}^{2}
$$

where the reference radius $R_{0}$ itself is a function increasing with the pressure $p$. Inequality (49) shows that given an internal pressure, the $F_{\mathrm{c}}$ value obtained from (48) must not be too high for the buckling solution to be meaningful. If the compressive force is greater than the upper bound specified by (49), the inflated beam collapses by crushing rather than by bending buckling. Thus, the bound given by (49) will be referred to as the crushing force of the inflated beam. 


\subsection{Remark}

If the term $F^{2} \Omega^{2} I_{0} / S_{0}$ in relation (48) is assumed a priori to be negligible, the following expression gives a good approximation for the critical force:

$$
F_{\mathrm{c}} \approx \frac{\left(E+P / S_{0}\right) I_{0} \Omega^{2}}{1+\Omega^{2} \frac{I_{0}}{S_{0}}+\Omega^{2} \frac{\left(E+P / S_{0}\right) I_{0}}{P+k G S_{0}}}
$$

By making $P \quad 0$ in the previous relation, one finds the well-known expression for the critical force of a classical beam, excepted the term $\Omega^{2} I_{0} / S_{0}$ in the denominator of (50). However, the pressure cannot vanish in the inflated beam according to the existence of the crushing force mentioned in (49). Now, by making the pressure grow to infinity, relation (50) provides an infinite buckling force, as expected.

Although the approximation (50) gives $F_{\mathrm{c}}$ values close to those given by (48) for usual numerical data, it will not be used in the following. Rather, the buckling force will be computed by means of the complete Relation (48).

\subsection{Numerical results}

The numerical computation is carried out with the same data as in Table 1 for the bending case. Table 4 and Fig. 4 give the buckling force $F_{\mathrm{c}}$ as a function of the length of the beam and the internal pressure, compared with the membrane finite element solution. As in Table 2 , the dimensions $\left(l_{0}, R_{0}, h_{0}\right)$ defining the reference configuration of the beam must be taken as those when the beam is pressurized.

According to the discussion on the validity of the solution, any critical force given by the inflated beam theory and greater than the resultant of the pressure on the ends $P \quad p \pi R_{0}^{2}$ must be rejected. In Table 4, these unacceptable values are marked out with a cross. As for the membrane finite element computation, it does correctly detect the crushing forces as bifurcation points. However, it then fails to determine the bifurcation mode, which would mean that the beam is indeed in ultimate collapse.

As shown in Table 4, the values for the buckling force obtained by the beam theory are in good agreement with that obtained with the membrane finite element. Over the whole range of the computation, the differences are less than $1 \%$. The pressurecrushing force curves given by relation (49) are shown in Fig. 4 in dashed lines. Since reference radius $R_{0}$ varies little with the pressure (see Table 4), these curves are almost straight lines. If the critical force $F_{\mathrm{c}}$ is found lower than these curves, then the beam buckles at this value. If not, the beam is crushed down before bending buckling occurs. As said above, the membrane finite element computation is able to correctly detect the crushing forces, and yields pressure-critical force curves stemming from the crushing curves, as expected. However, for the sake of clarity, these portions of curves are not represented in Fig. 4. 
Table 4

Critical loads $F_{\mathrm{c}}$ of a cantilever inflated tube

\begin{tabular}{|c|c|c|c|c|c|c|c|}
\hline \multirow{2}{*}{$\begin{array}{l}\text { Natural } \\
\text { length, } \\
l_{\varnothing}(\mathrm{m})\end{array}$} & \multirow{2}{*}{$\begin{array}{l}\text { Pressure } \\
\left(\mathrm{N} / \mathrm{m}^{2}\right)\end{array}$} & \multirow{2}{*}{$\begin{array}{l}\text { Reference } \\
\text { length, } l_{0}(\mathrm{~m})\end{array}$} & \multirow{2}{*}{$\begin{array}{l}\text { Reference } \\
\text { radius, } R_{0}(\mathrm{~m})\end{array}$} & \multirow{2}{*}{$\begin{array}{l}\text { Reference } \\
\text { thickness, } h_{0} \\
\text { (m) }\end{array}$} & \multicolumn{2}{|c|}{ Critical force $(\mathrm{N})$} & \multirow{2}{*}{$\begin{array}{l}\text { Diff- } \\
\text { eren- } \\
\text { ce } \\
(\%)\end{array}$} \\
\hline & & & & & $\begin{array}{l}\text { By the inflat- } \\
\text { able beam } \\
\text { theory }\end{array}$ & $\begin{array}{l}\text { By the mem- } \\
\text { brane finite } \\
\text { element }\end{array}$ & \\
\hline \multicolumn{8}{|c|}{ Case 1. Natural radius $R_{\varnothing}=0.04 \mathrm{~m}$} \\
\hline \multirow[t]{4}{*}{0.65} & $5.0 \times 10^{4}$ & $6.508 \times 10^{-1}$ & $4.022 \times 10^{-2}$ & $1.246 \times 10^{-4}$ & $x$ & $2.538 \times 10^{+2}$ & $x$ \\
\hline & $1.0 \times 10^{5}$ & $6.517 \times 10^{-1}$ & $4.044 \times 10^{-2}$ & $1.243 \times 10^{-4}$ & $3.669 \times 10^{+2}$ & $3.661 \times 10^{+2}$ & 0.2 \\
\hline & $1.5 \times 10^{5}$ & $6.525 \times 10^{-1}$ & $4.065 \times 10^{-2}$ & $1.239 \times 10^{-4}$ & $3.720 \times 10^{+2}$ & $3.714 \times 10^{+2}$ & 0.2 \\
\hline & $2.0 \times 10^{5}$ & $6.533 \times 10^{-1}$ & $4.087 \times 10^{-2}$ & $1.236 \times 10^{-4}$ & $3.773 \times 10^{+2}$ & $3.772 \times 10^{+2}$ & 0.0 \\
\hline \multirow[t]{4}{*}{0.9} & $5.0 \times 10^{4}$ & $9.012 \times 10^{-1}$ & $4.022 \times 10^{-2}$ & $1.246 \times 10^{-4}$ & $1.912 \times 10^{+2}$ & $1.911 \times 10^{+2}$ & 0.1 \\
\hline & $1.0 \times 10^{5}$ & $9.023 \times 10^{-1}$ & $4.044 \times 10^{-2}$ & $1.243 \times 10^{-4}$ & $1.939 \times 10^{+2}$ & $1.937 \times 10^{+2}$ & 0.1 \\
\hline & $1.5 \times 10^{5}$ & $9.035 \times 10^{-1}$ & $4.065 \times 10^{-2}$ & $1.239 \times 10^{-4}$ & $1.967 \times 10^{+2}$ & $1.968 \times 10^{+2}$ & 0.1 \\
\hline & $2.0 \times 10^{5}$ & $9.046 \times 10^{-1}$ & $4.087 \times 10^{-2}$ & $1.236 \times 10^{-4}$ & $1.994 \times 10^{+2}$ & $2.001 \times 10^{+2}$ & 0.3 \\
\hline \multirow[t]{4}{*}{1.15} & $5.0 \times 10^{4}$ & 1.151 & $4.022 \times 10^{-2}$ & $1.246 \times 10^{-4}$ & $1.178 \times 10^{+2}$ & $1.177 \times 10^{+2}$ & 0.1 \\
\hline & $1.0 \times 10^{5}$ & 1.153 & $4.044 \times 10^{-2}$ & $1.243 \times 10^{-4}$ & $1.195 \times 10^{+2}$ & $1.195 \times 10^{+2}$ & 0.0 \\
\hline & $1.5 \times 10^{5}$ & 1.154 & $4.065 \times 10^{-2}$ & $1.239 \times 10^{-4}$ & $1.211 \times 10^{+2}$ & $1.215 \times 10^{+2}$ & 0.3 \\
\hline & $2.0 \times 10^{5}$ & 1.156 & $4.087 \times 10^{-2}$ & $1.236 \times 10^{-4}$ & $1.228 \times 10^{+2}$ & $1.239 \times 10^{+2}$ & 0.9 \\
\hline \multicolumn{8}{|c|}{ Case 2. Natural radius $R_{\varnothing}=0.06 \mathrm{~m}$} \\
\hline \multirow[t]{4}{*}{0.65} & $5.0 \times 10^{4}$ & $6.512 \times 10^{-1}$ & $6.049 \times 10^{-2}$ & $1.245 \times 10^{-4}$ & $\times$ & $5.692 \times 10^{+2}$ & $x$ \\
\hline & $1.0 \times 10^{5}$ & $6.525 \times 10^{-1}$ & $6.098 \times 10^{-2}$ & $1.239 \times 10^{-4}$ & $\times$ & $1.141 \times 10^{+3}$ & $x$ \\
\hline & $1.5 \times 10^{5}$ & $6.537 \times 10^{-1}$ & $6.147 \times 10^{-2}$ & $1.234 \times 10^{-4}$ & $1.240 \times 10^{+3}$ & $1.238 \times 10^{+3}$ & 0.1 \\
\hline & $2.0 \times 10^{5}$ & $6.550 \times 10^{-1}$ & $6.196 \times 10^{-2}$ & $1.228 \times 10^{-4}$ & $1.266 \times 10^{+3}$ & $1.267 \times 10^{+3}$ & 0.1 \\
\hline \multirow[t]{4}{*}{0.9} & $5.0 \times 10^{4}$ & $9.017 \times 10^{-1}$ & $6.049 \times 10^{-2}$ & $1.245 \times 10^{-4}$ & $\times$ & $5.694 \times 10^{+2}$ & $x$ \\
\hline & $1.0 \times 10^{5}$ & $9.035 \times 10^{-1}$ & $6.098 \times 10^{-2}$ & $1.239 \times 10^{-4}$ & $6.518 \times 10^{+2}$ & $6.502 \times 10^{+2}$ & 0.2 \\
\hline & $1.5 \times 10^{5}$ & $9.052 \times 10^{-1}$ & $6.147 \times 10^{-2}$ & $1.234 \times 10^{-4}$ & $6.656 \times 10^{+2}$ & $6.655 \times 10^{+2}$ & 0.0 \\
\hline & $2.0 \times 10^{5}$ & $9.069 \times 10^{-1}$ & $6.196 \times 10^{-2}$ & $1.228 \times 10^{-4}$ & $6.797 \times 10^{+2}$ & $6.834 \times 10^{+2}$ & 0.5 \\
\hline \multirow[t]{4}{*}{1.15} & $5.0 \times 10^{4}$ & 1.152 & $6.049 \times 10^{-2}$ & $1.245 \times 10^{-4}$ & $3.959 \times 10^{+2}$ & $3.952 \times 10^{+2}$ & 0.2 \\
\hline & $1.0 \times 10^{5}$ & 1.154 & $6.098 \times 10^{-2}$ & $1.239 \times 10^{-4}$ & $4.043 \times 10^{+2}$ & $4.039 \times 10^{+2}$ & 0.1 \\
\hline & $1.5 \times 10^{5}$ & 1.157 & $6.147 \times 10^{-2}$ & $1.234 \times 10^{-4}$ & $4.128 \times 10^{+2}$ & $4.142 \times 10^{+2}$ & 0.3 \\
\hline & $2.0 \times 10^{5}$ & 1.159 & $6.196 \times 10^{-2}$ & $1.228 \times 10^{-4}$ & $4.215 \times 10^{+2}$ & $4.252 \times 10^{+2}$ & 0.9 \\
\hline \multicolumn{8}{|c|}{ Case 3. Natural radius $R_{\varnothing}=0.08 \mathrm{~m}$} \\
\hline \multirow[t]{4}{*}{0.65} & $5.00 \times 10^{4}$ & $6.517 \times 10^{-1}$ & $8.087 \times 10^{-2}$ & $1.243 \times 10^{-4}$ & $\times$ & $1.011 \times 10^{+3}$ & $x$ \\
\hline & $1.00 \times 10^{5}$ & $6.533 \times 10^{-1}$ & $8.174 \times 10^{-2}$ & $1.236 \times 10^{-4}$ & $\times$ & $2.025 \times 10^{+3}$ & $\times$ \\
\hline & $1.50 \times 10^{5}$ & $6.550 \times 10^{-1}$ & $8.261 \times 10^{-2}$ & $1.228 \times 10^{-4}$ & $2.870 \times 10^{+3}$ & $2.886 \times 10^{+3}$ & 0.6 \\
\hline & $2.00 \times 10^{5}$ & $6.567 \times 10^{-1}$ & $8.348 \times 10^{-2}$ & $1.221 \times 10^{-4}$ & $2.951 \times 10^{+3}$ & $2.954 \times 10^{+3}$ & 0.1 \\
\hline \multirow[t]{4}{*}{0.9} & $5.00 \times 10^{4}$ & $9.023 \times 10^{-1}$ & $8.087 \times 10^{-2}$ & $1.243 \times 10^{-4}$ & $x$ & $1.011 \times 10^{+3}$ & $\times$ \\
\hline & $1.00 \times 10^{5}$ & $9.046 \times 10^{-1}$ & $8.174 \times 10^{-2}$ & $1.236 \times 10^{-4}$ & $1.529 \times 10^{+3}$ & $1.525 \times 10^{+3}$ & 0.2 \\
\hline & $1.50 \times 10^{5}$ & $9.069 \times 10^{-1}$ & $8.261 \times 10^{-2}$ & $1.228 \times 10^{-4}$ & $1.572 \times 10^{+3}$ & $1.571 \times 10^{+3}$ & 0.0 \\
\hline & $2.00 \times 10^{5}$ & $9.092 \times 10^{-1}$ & $8.348 \times 10^{-2}$ & $1.221 \times 10^{-4}$ & $1.616 \times 10^{+3}$ & $1.625 \times 10^{+3}$ & 0.5 \\
\hline \multirow[t]{4}{*}{1.15} & $5.00 \times 10^{4}$ & 1.153 & $8.087 \times 10^{-2}$ & $1.243 \times 10^{-4}$ & $9.304 \times 10^{+2}$ & $9.312 \times 10^{+2}$ & 0.1 \\
\hline & $1.00 \times 10^{5}$ & 1.156 & $8.174 \times 10^{-2}$ & $1.236 \times 10^{-4}$ & $9.569 \times 10^{+2}$ & $9.553 \times 10^{+2}$ & 0.2 \\
\hline & $1.50 \times 10^{5}$ & 1.159 & $8.261 \times 10^{-2}$ & $1.228 \times 10^{-4}$ & $9.840 \times 10^{+2}$ & $9.869 \times 10^{+2}$ & 0.3 \\
\hline & $2.00 \times 10^{5}$ & 1.162 & $8.348 \times 10^{-2}$ & $1.221 \times 10^{-4}$ & $1.012 \times 10^{+3}$ & $1.021 \times 10^{+3}$ & 1.0 \\
\hline
\end{tabular}

The buckling force $F_{\mathrm{c}}$ decreases as the tube length increases, and it increases along with the internal pressure. With $R_{\varnothing} \quad 0.08 \mathrm{~m}$, for instance, $F_{\mathrm{c}}$ is divided by about three from $l_{\varnothing} \quad 0.65$ to $1.15 \mathrm{~m}$. With $R_{\varnothing} \quad 0.08 \mathrm{~m}$ and $l_{\varnothing} \quad 1.15 \mathrm{~m}, F_{\mathrm{c}}$ rises by about $10 \%$ when the pressure varies from $0.5 \times 10^{5}$ to $2 \times 10^{5} \mathrm{~N} / \mathrm{m}^{2}$. Other computations, which are not 

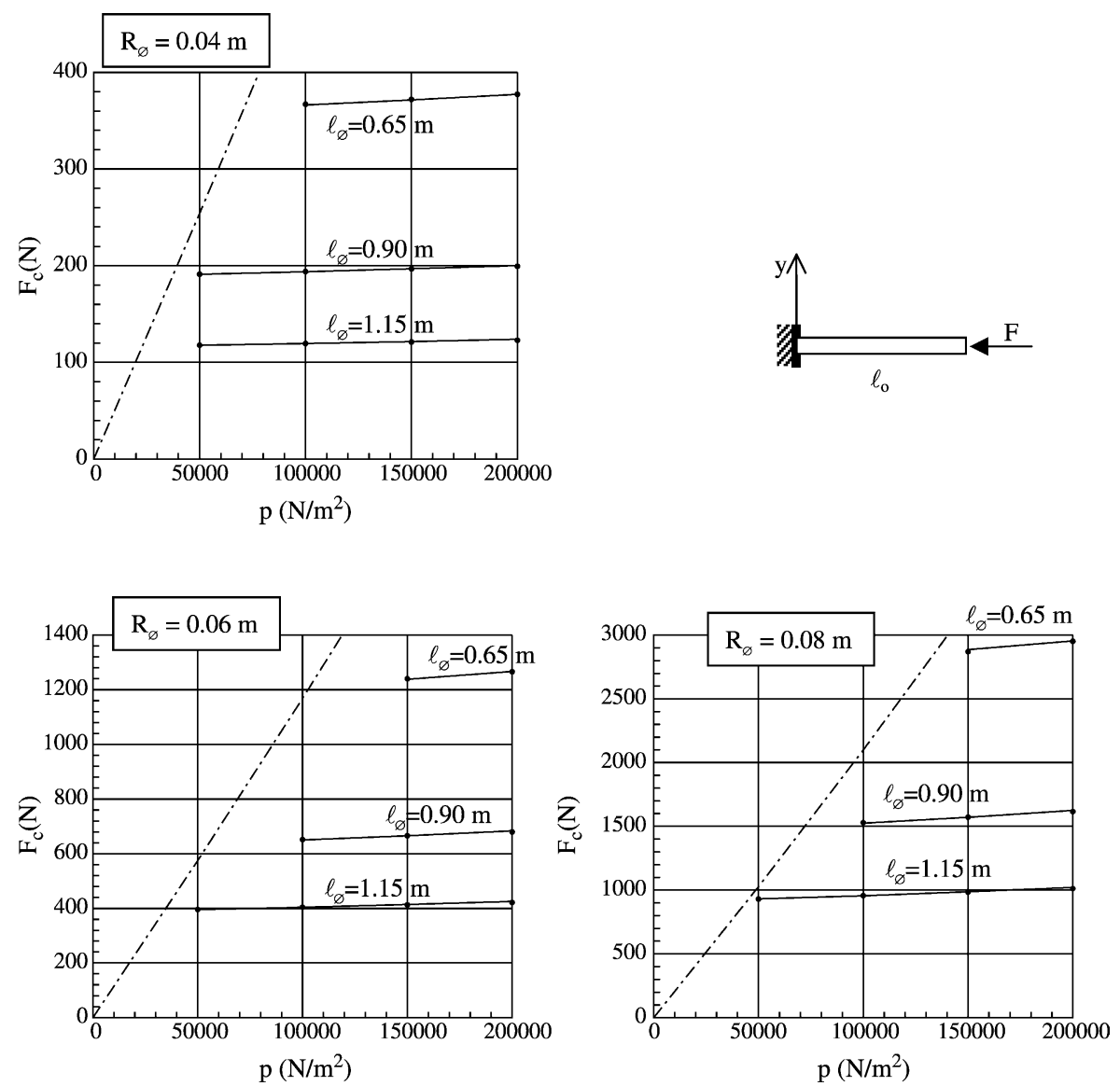

Fig. 4. Critical loads of a cantilever. (•) inflatable beam theory, ( ) membrane finite element, ( • • ) crushing force.

presented here, show that the influence of the pressure on the buckling force is stronger if a material with lower Young modulus is chosen. Nevertheless, the strains can then be so large that a fully non-linear computation is required.

If the pressure were not taken into account, the discrepancy between the beam theory and the membrane finite element computation should reach $4.1 \%$. Furthermore, if the correction coefficient $k$ is given very large values (one then reduces to then Euler Bernoulli model), then the discrepancy reaches $13.4 \%$.

\section{Conclusions}

The non-linear equations have been derived for the in-plane stretching and bending of an inflated Timoshenko beam undergoing finite rotations. The corresponding linearized equations have been obtained and then analytically solved for the bending 
and the buckling cases. New theoretical and numerical results on the buckling of inflatable beams are displayed. The following facts have been emphasized.

(i) It is crucial to distinguish between the so-called natural configuration where the internal pressure is zero and the pre-stressed reference configuration around which the linearization is performed. The dimensions defining the reference configuration of the beam depend on the prescribed internal pressure.

(ii) The analytical solutions have clearly shown the beneficial effects of the internal pressure on the bearing capacity of the beam: the inflation amounts to increasing the material properties.

(iii) The analytical solution for the bent beam only holds if the pressure is greater the so-called wrinkling pressure. Similarly, the solution for the buckled beam is valid only if the compressive force is less than the so-called crushing force.

The numerical computations have been performed on nine beam geometries (three values of natural radius by three values of natural length) and four values of the internal pressure. The maximum deflections or the buckling forces have been found to be in good agreement with the membrane finite element values obtained within the three-dimensional framework.

Further investigations are in progress in order to obtain analytical solutions in dynamics and derive finite elements for solving complex geometries and loadings of inflated beams.

\section{References}

[1] Comer RL, Levy S. Deflections of an inflated circular cylindrical cantilever beam. AIAA J 1963;1(7):1652 5.

[2] Fichter WB. A theory for inflated thin wall cylindrical beams. NASA TN D 3466; 1966.

[3] Main A, Peterson SW, Strauss AM. Load deflection behaviour of space based inflatable fabric beams. J Aerospace Eng 1994;2(7):225 38.

[4] Wielgosz C, Thomas JC. Deflections of inflatable fabric panels at high pressure. Thin Walled Struct 2002;40: 52336.

[5] Thomas JC, Wielgosz C. Deflections of highly inflated fabric tubes. Thin Walled Struct 2004;42:1049 66.

[6] Wielgosz C, Thomas JC. An inflatable fabric beam finite element. Commun Numer Meth Eng 2003;19: 30712.

[7] Cowper GR. The shear coefficient in Timoshenko's beam theory. J Appl Mech 1967;33:335 40. 